\title{
LE DISCOURS ET SES SITUATIONS
}

Abstract. Rey-Debove Josette, Le discours et ses situations [Discourse in various contexts]. Studia Romanica Posnaniensia, Adam Mickiewicz University Press. Poznań, vol. XXV/XXVI: 2000, pp. 261268, ISBN 83-232-0965-0, ISSN 0137-2475.

This paper aims to situate the various areas of discourse analysis with respect to general typological or intersecting characteristics, and to extend our apprehension of «true meaning» as far as possible.

It suggests that unacceptable phrases and paralinguistic elements can be included in semantics, and that non-spontaneous cited or transcribed discourse can be considered alongside spontaneous oral and written discourse.

A distinction is made between the "producer" (who is «responsible») and the «emitter»; between the "addressee» (who is deliberately targeted) and the «receiver». The emitter is often citing a third party, while the addressee is often non-specific or accidental.

Provision is made for conflictual relationships between speakers (claiming the right to speak in public) and messages with pseudo addressees, which give the function of expression priority over the function of communication. The phatic function merely maintains a relationship between speakers which is detrimental to meaning, just as violence may ensue when intercomprehension breaks down.

\section{ESSOR DE L'ÉTUDE DU DISCOURS}

En France, la recherche sur le discours est très active; elle est liée aux changements de point de vue sur la langue qui sont devenus nécessaires pour accéder au sens tel que nous l'expérimentons. L'étude pure et dure du langage comme système immanent fut fondamentale pour constituer la linguistique comme science; mais la pauvreté des résultats menait à une impasse prévisible. Nous ne pouvons plus concevoir une langue comme une grammaire qui met en œuvre un lexique, nous ne croyons plus à la phrase comme unité de travail. Une approche sémiotique - que personnellement nous défendons depuis les années 70 - est devenue indispensable, approche qui tient compte d'une théorie générale des signes, de la sociologie, de la psychologie et de la pragmatique. Autrement nous n'avons aucun accès au sens réel, et le sens est la raison d'être du langage, tout le reste étant secondaire. 


\section{ÉLOGE DU GLOBALISME}

Je me permettrai une opinion personnelle sur la recherche en sciences humaines. Comme ce sont des sciences dites «molles», on fait porter les efforts d'analyse sur ce qui semble constituer un «noyau dur», que l'on espère étoffer par la suite. Or, je suis persuadée que c'est une erreur de méthode de ne pas admettre d'abord une approche globaliste afin de retrouver, plus tard, un noyau dur et des règles infalsifiables. J'ai expérimenté moi-même que c'est l'étude du métalangage (langage second et secondaire si l'on veut) qui m'a permis d'avoir une notion plus claire du langage. Évidemment il est plus difficile de rechercher les limites d'un domaine que son centre, dans un ensemble flou et pluridisciplinaire. Mais il fallait débloquer la situation crée par la grammaire générative et la sémantique des traits différentiels.

\section{ENTRE LANGUE ET EXPRESSION INDIVIDUELLE}

Est-ce à dire que l'on va passer brutalement de l'étude de la langue à l'analyse véritable d'un discours particulier? Personnellement, en tant que linguiste, c'est le discours en général qui m'intéresse le plus, ce que dans son schéma Systema, norma y habla, Coseriu désigne par norma, c'est-à-dire la parole collective basée sur un consensus social ou pour Klaus Heger la sigma parole, ou, pour le linguiste américain Hocket, l'intersection des idiolectes. Mais l'intersection des idiolectes se calcule sur la base des idiolectes. Le discours personnel n'est nullement exclu; j'ai moi-même fait une analyse d'un manuscrit de Proust, article intitulé «Pour une lecture de la rature», qui a une portée générale pour la production du texte.

Sur l'ensemble des problèmes concernant l'analyse du discours en France, je vous renvoie au numéro 117 de la revue Langages (Larousse) dirigé par Dominique Maingueneau et paru en 1995, qui en donne une idée assez fidèle. L'analyse du discours dépasse de beaucoup le champ du Discourse Analysis américain, centré sur l'oral, le dialogue et les interactions sociales. Les Français s'intéressent à l'oral et à l'écrit, étudient la notion de corpus, de didacticité, de reformulation, de contrat de communication, de destinataire différé. Le rôle du contexte, de la situation et des paralangages est toujours pris en compte dans les typologies des discours qu'ils proposent.

Je tenterai ici de donner un point de vue encore plus général et globaliste, espérant ne rien oublier d'important, sans toutefois entrer dans le détail, car ce serait l'objet d'un livre.

\section{DISTINCTIONS ET PRÉCISIONS TERMINOLOGIQUES}

D'abord je dois préciser l'emploi de quelques termes; j'appelle discours un acte langagier oral ou écrit, qui peut être particulier ou correspondre à un genre collectif, qui a un destinataire immédiat ou différé; ce destinataire pouvant être multiple et 
pouvant aussi être la même personne que le producteur du discours. Patrick Charaudeau, dans son excellent article de sémiolinguistique définit le discours comme «contrat de communication» mais ce contrat ne concerne pour moi que le dialogue. Par discours, j'entends ce qui est oral (parole) et ce qui est écrit (le texte), mais en cas d'ambiguilté, il est préférable de réserver discours pour l'oral et texte pour l'écrit.

Le discours englobe l'énonciation qui se caractérise par la présence de déictiques (ego, hic et nunc), possède toujours un deixis. Le discours englobe aussi le dialogue mot qui étymologiquement signifie "parler en travers, de l'un à l'autre» (c'est le diade diagonale) et qui est compris aujourd'hui comme "parler à deux»; il est donc plus prudent de dire plurilogue quand il y a plus de deux personnes. Le dialogue englobe la conversation ou «échange de propos, naturel et spontané» selon le Petit Robert; elle était autrefois uniquement orale, mais se réalise aujourd'hui par écrit, en internet.

\section{PHRASES INACCEPTABLES ET MIXTES}

Pour les caractères internes du discours, on doit récupérer deux productions courantes généralement écartées 1) production des phrases inacceptables (agrammaticales et/ou asémantiques), parce que ces phrases véhiculent du sens, ou au moins du contenu (par exemple le «petit nègre», «les délires», les «bribes» de Claire Blanche-Benveniste, les «transgressions» du poète); ainsi que les écrits non linéaires comme les tableaux et parfois les publicités, parce que leur lecture est différente mais possible. 2) production des énoncés mixtes et les 3 types importants de mixité: mixité des systèmes sémiotiques (par exemple les chiffres, les signes mathématiques, les symboles chimiques etc.) ainsi que la mixité oral/écrit (explication orale du professeur qui écrit au tableau). Également la mixité du discours métalinguistique qui est capable de produire des autonymes dans toutes les langues, seul type de babélien tout à fait correct (exemple, le dictionnaire étymologique de Walter von Wartburg).

\section{PARALANGAGES}

Pour les caractères externes du discours, on devra bien sûr tenir compte des langages d'accompagnement ou paralangages; à l'oral essentiellement de la mimique et de la gestuelle, à l'écrit imprimé la mise en page, la typographie porteuse de sens, la présence de l'image et de la couleur. On considère que l'intonation et les pauses appartiennent au langage oral, tout comme la ponctuation au sens large appartient au langage écrit. 


\section{ÉMETTEUR/RÉCEPTEUR ET PRODUCTEUR/DESTINATAIRE}

Je crois également utile de distinguer le couple émetteur/récepteur du couple producteur/destinataire, seul le second couple étant personnellement et directement impliqué. Dans cette optique, le comédien au thétre ou l'interprète de chansons n'est qu'un émetteur, et celui qui entend la dispute de ses voisins n'est qu'un récepteur. L'interprète qui traduit dans un échange à trois actants est un récepteur-transcodeur-émetteur.

\section{CONTEXTE ET SITUATION}

Enfin on séparera soigneusement le contexte langagier qu'on appellera contexte, du contexte situationnel, qu'on appellera situation. La dimension que l'on donne à un discours peut tolérer un contexte transphrastique, présent ou différé (autres discours du même producteur). Ainsi, un récepteur pourra s'étonner d'un discours qui ne «correspond pas au personnage» (politique, ou plus intime). Le contexte langagier est inégalement pertinent au niveau transphrastique. Au contraire la situation constitue presque toujours un apport de sens ou une désambiguïsation. La situation c'est l'espace-temps des actants qui participent activement ou passivement à un discours, ces actants pouvant être l'émetteur et le récepteur, ou bien le producteur et le destinataire, ou même un mixage des actants des deux couples (par exemple, récepteur qui lit frauduleusement une lettre destinée à quelqu'un d'autre).

\section{GASPILLAGE DES DISCOURS PARTICULIERS}

La situation d'un discours particulier est quasiment impossible à connaître réellement. Elle est faite de présomptions sur ce qui est pertinent pour le lieu, le temps et les actants. Ce sont les actants qui posent le plus de problèmes: quelles sont leurs relations interpersonnelles, leurs rôles respectifs, quel est leur savoir partagé, quel type d'action veulent-ils exercer? De quoi est faite leur mémoire? Ainsi le fameux Rose Bud prononcé par Citizen Kane en mourant, et que personne ne put jamais interpréter sauf lui-même (nom de son traîneau d'enfant). La situation ajoute toujours du sens à un énoncé, mais elle gaspille la plupart des discours qui ne seront jamais décryptés.

\section{COMPOSANTES FONDAMENTALES DES SITUATIONS DE DISCOURS}

Être certaine de pouvoir les hiérarchiser, dans cette présentation provisoire. Du point de vue de l'analyse du sens, c'est l'opposition oral/écrit qui domine, mais du point de vue de la diffusion du sens, c'est l'opposition entre le discours privé et le 
discours de ceux qui ont droit à l'expression publique, orale ou écrite. Un autre axe généralement négligé me semble très important, c'est l'opposition discours spontané/discours repris, qui met en jeu soit une reprise de son propre discours (texte oralisé, ou discours transcrit) soit la reprise du discours d'un producteur par un émetteur différent.

\section{DISCOURS TRANSCRIT, TEXTE ORALISÉ}

Dans le premier cas, la traditionnelle opposition oral/écrit se déploie en quatre modes d'expression: oral spontané/écrit oralisé - écrit spontané/oral transcrit. On n'est jamais certain d'avoir affaire à l'oral ou à l'écrit, à cause des modes non spontanés, dont les origines scolaires de transcodage formel sont bien connues. C'est la dictée pour la transcription, et la lecture pour l'oralisation. Le développement des médias a multiplié les discours non spontanés: discours appris par cœur, lecture du texte du prompteur (ou souffleur) qui se déroule devant celui qui parle. Mais en principe, cette promenade dans les quatre modes d'expression conserve le producteur originel.

\section{DISCOURS CITÉ}

Il est tout autrement dans le second cas, où le producteur n'est pas l'émetteur, mais une autre personne dont le discours ou le texte est cité par l'émetteur. C'est alors la responsabilité qui entre en jeu, avec la distinction des personnes qui sont producteurs et émetteurs. Le producteur est responsable de son discours ou de son texte, l'émetteur ne l'est pas. Le citeur est responsable du choix de son discours rapporté (celui qui dit le Pater Noster est responsable d'avoir choisi de le dire; le lexicographe qui cite un écrivain antisémite est responsable du choix de la citation). Le langage étant de nature sociale, la notion de responsabilité du contenu ne peut être évacuée, dans la mesure ou tout discours enferme une possibilité d'erreur, de mensonge et d'incitation à la violence.

\section{L'ORAL LIEU DES CONTACTS ET DES SANCTIONS}

C'est dans l'opposition oral/écrit que se manifeste le mieux la communication directe ou différée. L'oral est le plus souvent immédiat, que la parole soit privée ou publique, et l'écrit est le plus souvent différé dans tous les cas.

L'oral se signale par l'importance de la présence physique du destinataire, même partielle comme au téléphone; mais la situation la plus signifiante à cause des paralangages est celle où l'on voit le locuteur; il est très différent de dialoguer face à face ou d'une pièce à l'autre sans se voir, situation plus proche de celle du téléphone. On sait aussi que le message verbo-visuel de la télévision est plus dangereux pour le producteur du discours que le message uniquement sonore de la radio. 


\section{DIALOGUE AVEC PARTENAIRE}

L'oral est essentiellement le lieu du dialogue, une situation où le producteur attend un échange, une réponse. Même le silence de l'interlocuteur est un type de réponse. Cet échange véritable est un lieu d'initiative, de prise de parole, de combat verbal qui ont été bien étudiés.

Je ne m'y attarderai pas, sauf pour souligner la responsabilité partagée des producteurs et la possibilité constante de sanctions tant positives que négatives entre partenaires (par exemple: «je suis d'accord, vous vous trompez, vous déguisez la vérité, vous n'êtes pas compétent etc.»). Relèvent de la situation dialogique le débat public, l'interview, la conférence suivie de questions, l'interrogation scolaire, l'interrogatoire juridique. Le cas-limite du dialogue se présente quand le rapport de force entre partenaires est trop inégal; situation sociale facile à prévoir dans une typologie. Si les actants ne se craignent pas l'un l'autre et s'ils sont combatifs, un recouvrement des discours peut se produire, situation tout à fait contraire à l'expression orale qui ne supporte pas le cumul polyphonique dans l'instant. Ce cumul est évité par un tiers actant, sorte d'arbitre qui dirige les débats à la seconde près, parfois même ce rôle échoue à l'interprète, dans les dialogues télévisés.

\section{DIALOGUE SANS VRAI PARTENAIRE}

L'oral présente aussi des situations spécifiques quant au destinataire. C'est oralement qu'on est en présence de pseudo-destinataires: on se parle à soi-même, on s'adresse à son bébé sans s'occuper de la réception du message, tout comme on s'adresse à son chat ou à son chien. Cette situation est plus fréquente qu'on ne l'imagine; dans une société où l'information augmente et où la solitude s'accentue, on est obligé de s'exprimer sans réception possible. On parle même aux machines, généralement pour les injurier.

\section{LE DIALOGUE DES AUTRES: TRANSMISSION VERBO-VISUELLE}

La situation la plus courante du dialogue est directe par la présence et le son, et on peut y assister comme récepteur, sans être actant. Dès que cette situation est transmise par une technique quelconque, les récepteurs ne sont plus que des spectateurs. Déjà au théâtre, on nous propose le discours d'autres personnes en action, action dont nous sommes exclus. La situation est plus complexe dans la transmission par radio, cinéma et télévision, où les protagonistes sont absents: on les perçoit par le son, ou par l'image-son. Le son n'est pas toujours la parole, on sait l'importance de la musique de film comme paralangage. Quant à l'image elle nous montre parfois, dans les sous-titres, la traduction écrite de ce qui se dit, c'est-à-dire un transcodage double: passage de l'oral à l'écrit et passage d'une langue à une autre. Le film télévisé à sous-titres est un des discours les plus complexes, du point de vue sémiotique. 


\section{L'ÉCRIT, LIEU DU DIFFÉRÉ ET DE L'INDÉTERMINÉ}

L'écrit est le plus souvent différé; la lettre particulière envoyée à un destinataire en est déjà un bon exemple, et même la télécopie qui, par sa rapidité se rapproche du téléphone. Mais à cette notion de différé liée à la non-présence du destinataire, s'ajoute celle de l'indétermination fréquente du destinataire. Le cas extrême est le texte de l'écrivain, où la fonction d'expression prime souvent la fonction de communication, qui est beaucoup plus fruste.

\section{CIBLAGE DE L'ÉCRIT}

Mais entre ces deux cas se présentent tous les cas de ciblage de groupes sociaux plus ou moins déterminés; le journaliste connaît ses lecteurs, l'auteur de manuels scolaires aussi. Le chercheur écrit pour d'autres chercheurs avant que le récepteur-vulgarisateur ne serve de relais social. On ne sait pas toujours à qui s'adressent les publicités, et le cas devient obscur pour les graffitis et les tags. L'écrit se caractérise par la situation de non-réponse au producteur lorsque le destinataire est indéterminé, c'est-à-dire dans la plupart des cas.

\section{IMPORTANCE SOCIALE DU PRODUCTEUR}

Mais si le producteur a une surface sociale ou médiatique, il est aussitôt assiégé par un courrier postal désordonné auquel il ne répond pas. Ceci est vrai aussi du producteur oral de même profil, car la réponse écrite est toujours possible, alors que la réponse orale ne l'est pas du tout. Comment répondre à celui qui a parlé à des millions de récepteurs, sinon en lui écrivant? Faut-il dire que la parole publique est centrifuge et que le mouvement de réponse centripète ne peut s'effectuer que par l'écrit? On voit comment s'entrecroisent les axes des situations et le déplacement des critères d'importance.

\section{LE DISCOURS FONCTIONNEL ET LE DISCOURS PHATIQUE}

La relation des actants détermine aussi deux grands modes de communication, le fonctionnel et le phatique. Car personne ne s'exprimant pour ne rien dire - c'est une hypothèse de travail très répandue - les discours phatiques remplacent leur sens linguistique par un contenu affectif: ainsi la conversation décousue, les échanges de politesse, la carte postale de vacances ou la carte de vœux pour l'année nouvelle sont-elles centrées sur les actants; c'est une autre limite du discours où n'est retenue que l'intention de communiquer. 


\section{TYPOLOGIE FORMELLE DES ÉNONCÉS}

Je laisserai à d'autres chercheurs le soin de connecter les situations au types linguistiques formels d'énoncés, me contentant de mentionner: le discours du savoir (assertif, informatif, narratif, argumentatif), le discours de la demande (interrogatif, impératif, vocatif), le discours de l'action (déontique, performatif, agressif, séducteur). Le discours de l'action s'oriente, comme le discours phatique, vers la disparition du sens langagier au profit du comportement des actants, éventuellement jusqu'aux coups ou jusqu'aux caresses. Et ici on voit bien que mon exposé doit se terminer. 